\title{
Intraoperative Doppler echocardiography of pulmonary venous flow is useful in predicting reoperation in pulmonary artery banding
}

Keiko Bamba, Hidekazu Imai, Hiroshi Baba

Division of Anesthesiology, Niigata University Graduate School of Medical and Dental Sciences, Niigata, Japan

\section{Introduction}

- Bilateral pulmonary artery banding (PAB) is used to treat congenital heart disease in some high-risk neonates.

- Until recently, the appropriate PAB size depended on pulmonary artery pressures, partial pressure of oxygen in the arterial blood $\left(\mathrm{PaO}_{2}\right)$, and stable hemodynamics. However, high reoperation rates exist due to insufficient or excessive restriction of pulmonary blood flow.

- Pulmonary venous Doppler flow is an effective quantitative evaluation of pulmonary blood flow.

We hypothesized that the pulmonary venous velocity time integral (PV-VTI) in transesophageal echocardiography (TEE) during bilateral PAB surgery can predict the need for reoperation.

Figure 1: Before and after the PV VTI in the TEE pulsed doppler study
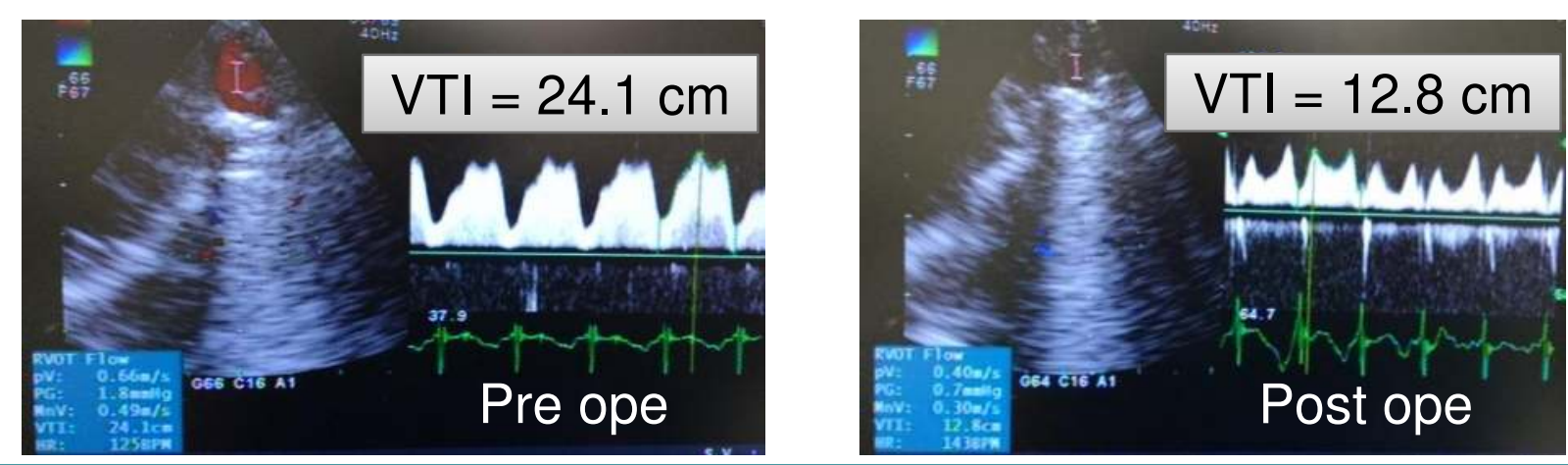

\section{Methods}

\section{Study population}

- Patients with congenital heart diseases underwent bilateral PAB at our hospital from January 2011 to September 2016.

\section{Study design}

- We investigated PV-VTI before PAB (pre-VTI) and after PAB (post-VTI) from the intraoperative TEE record.

- PV VTI was defined as the total value obtained by measuring the blood flow of four pulmonary veins.

- De-PAB group : Reoperation due to excessive PBF restriction.

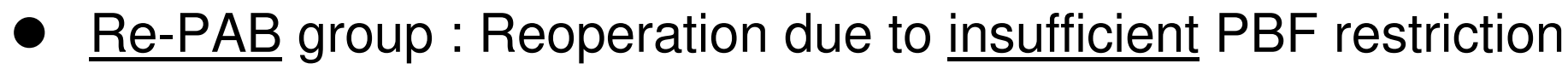

- Outcome was defined as reoperation within 3 months after the initial PAB surgery.

- We compared the two groups to a no-reoperation group.

- We retrospectively examined the association between the PVVTI ratio (post VTI / pre VTI) and incidence of reoperation.

\section{Statistical analysis}

- Comparisons were made with the Student's t-test and $p<0.05$ was considered significant.

- Data are expressed as mean \pm SD.

\section{Results}

Figure 2 : Study inclusion/exclusion flow diagram

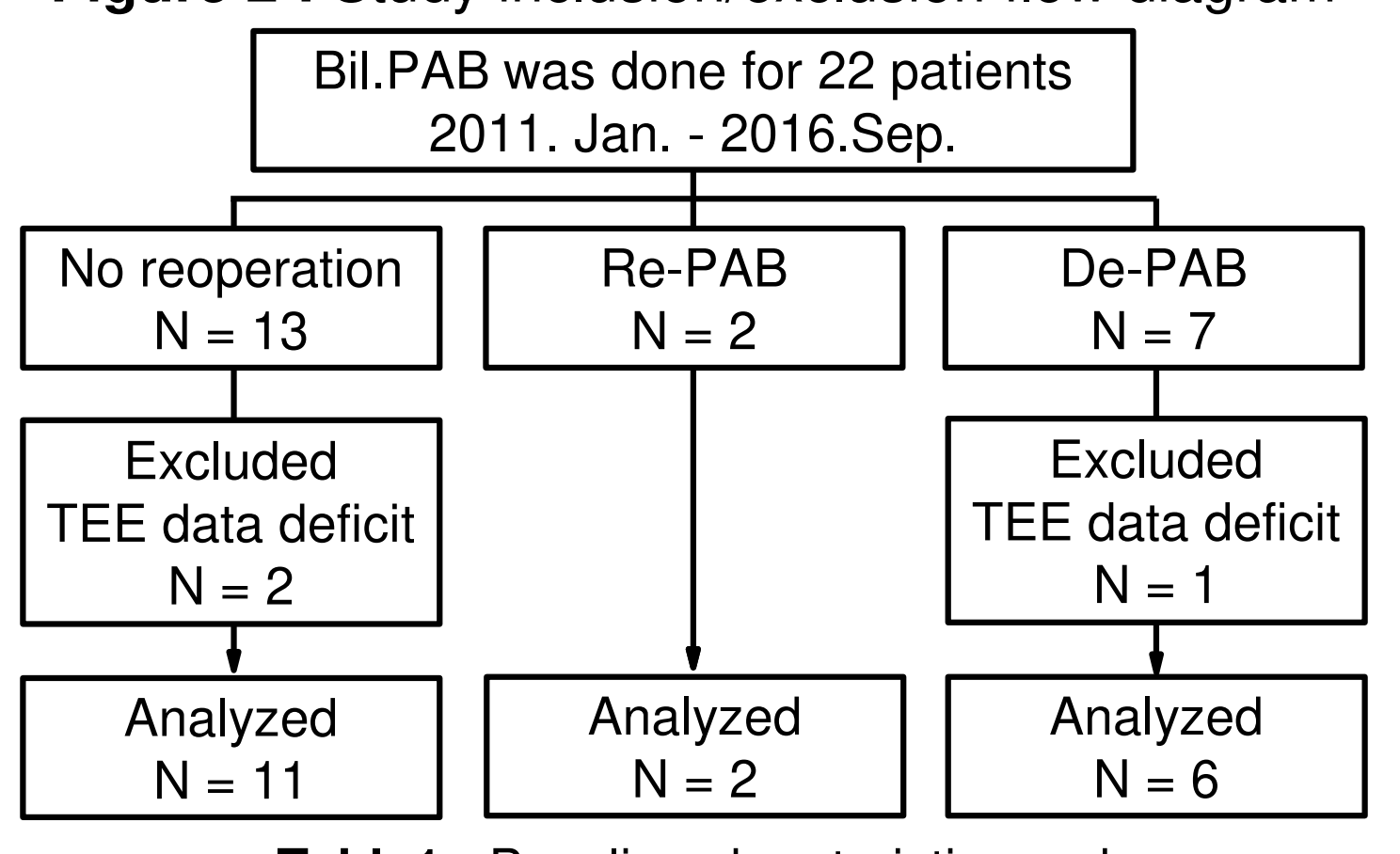

Table1 : Baseline charsteristics and

Intraoperative hemodynamic variables of patients

\begin{tabular}{|c|c|c|c|}
\hline & $\begin{array}{c}\text { No reoperations } \\
\qquad N=11\end{array}$ & $\begin{array}{c}\mathrm{Re}-\mathrm{PAB} \\
\mathrm{N}=2\end{array}$ & $\begin{array}{c}\text { De-PAB } \\
N=6\end{array}$ \\
\hline \multicolumn{4}{|l|}{ Charasteristics } \\
\hline Gender (Female/Male) & $5 / 9$ & $1 / 1$ & $4 / 2$ \\
\hline \multirow{2}{*}{ Age (days) } & 5.8 & 4.5 & 5.3 \\
\hline & $2-12$ & 2,7 & $2-8$ \\
\hline Body Weight $(\mathrm{kg})$ mean & 2.8 & 3.4 & 2.4 \\
\hline range & $2.0-3.5$ & $2.9,4.0$ & $1.8-3.6$ \\
\hline \multicolumn{4}{|l|}{ Diagnosis } \\
\hline AVSD & 1 & & \\
\hline DORV & 0 & & \\
\hline SRV & 1 & 1 & 2 \\
\hline CoA & 1 & & \\
\hline HLHS & 3 & & 2 \\
\hline MA & 1 & 1 & \\
\hline IAA & 3 & & 1 \\
\hline Truncus & 1 & & 1 \\
\hline \multicolumn{4}{|l|}{ Hemodynamic variables } \\
\hline $\mathrm{SaO}_{2}$ (post ope) (\%) & $84.8 \pm 10.1$ & $81.1 \pm 4.4$ & $77.3 \pm 2.9$ \\
\hline $\mathrm{PaO}_{2}$ (post ope) (mmHg) & $43.9 \pm 12.7$ & $43.0 \pm 4.0$ & $35.3 \pm 3.6$ \\
\hline Mean BP post-pre $(\mathrm{mmHg})$ & $12.9 \pm 7.8$ & $8.1 \pm 5.8$ & $7.5 \pm 7.8$ \\
\hline
\end{tabular}

AVSD, atrioventricular septal defect; DORV, double outlet right ventricle; SRV, single right ventricle; CoA, coarctation of the aorta; HLHS, hypoplastic left heart syndrome; MA, mitral atresia; IAA, interrupted aortic arch; Truncus, truncus arteriosus
Table2 : Bil. PAB PV VTI post/pre

\begin{tabular}{|cccccc|}
\hline PV VTI & $\begin{array}{c}\text { No reoperation } \\
\mathbf{N}=\mathbf{1 1}\end{array}$ & $\begin{array}{c}\mathbf{R e}-\mathbf{P A B} \\
\mathbf{N}=\mathbf{2}\end{array}$ & $\mathbf{P}$ & $\begin{array}{c}\text { De-PAB } \\
\mathbf{N}=\mathbf{6}\end{array}$ & $\mathrm{P}$ \\
\hline Pre VTI & $64.7 \pm 22.3$ & $60.5 \pm 23.9$ & 0.80 & $70.0 \pm 20.4$ & 0.63 \\
Post VTI & $36.5 \pm 10.7$ & $46.1 \pm 12.5$ & 0.28 & $29.7 \pm 10.7$ & 0.23 \\
PV VTI ratio & $0.57 \pm 0.12$ & $0.78 \pm 0.10$ & 0.05 & $0.41 \pm 0.07$ & 0.01 \\
\hline
\end{tabular}

Figure3 : PV VTI ratio

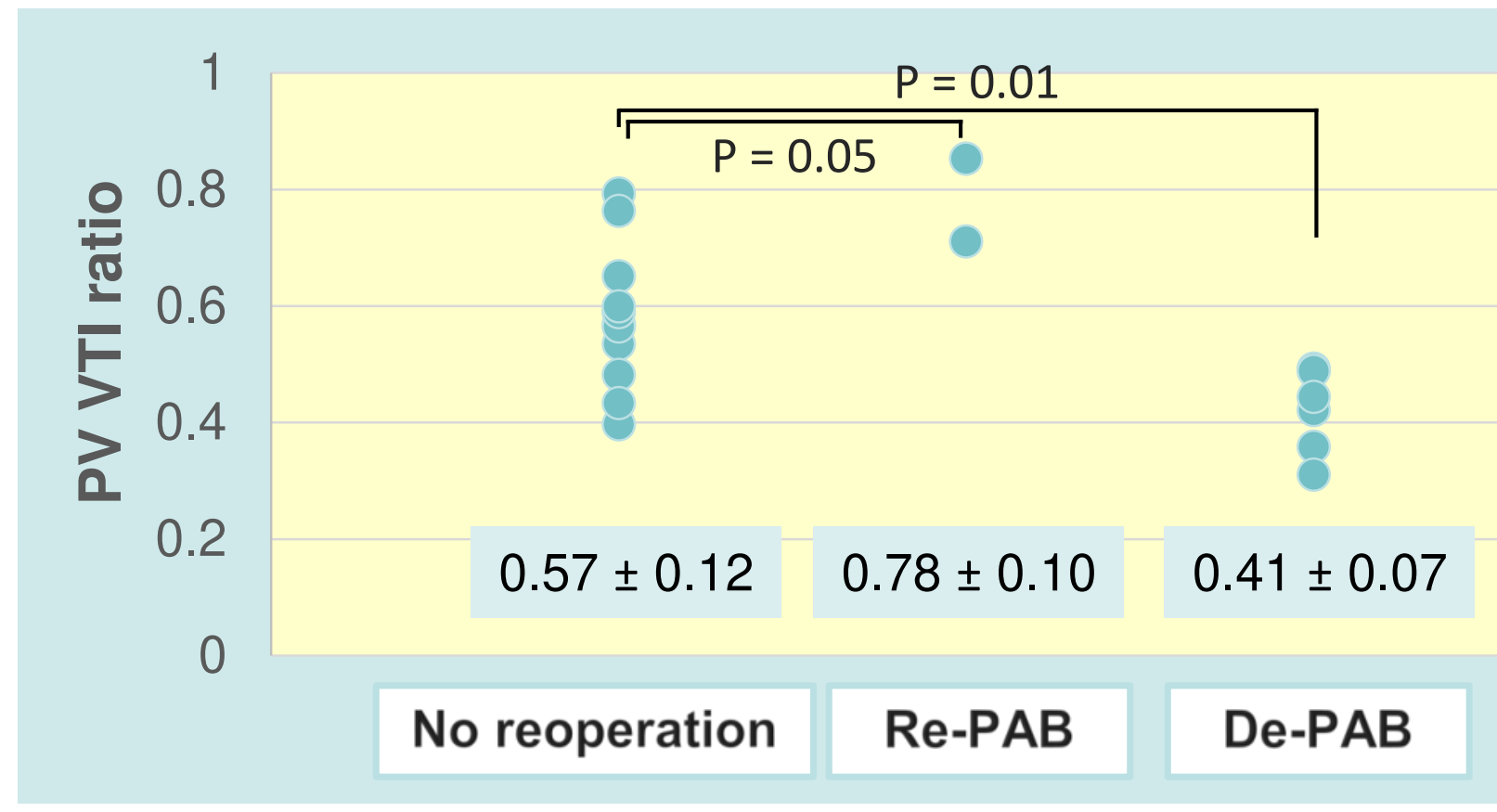

\section{Discussion}

- In the no reoperation group, the PV-VTI ratio showed an approximately $60 \%$.

- In the De-PAB group, the PV-VTI ratio was significantly reduced.

- In the Re-PAB group, the PV-VTI ratio showed a tendency to increase.

- This study suggests that the PV-VTI ratio may be useful to determine the optimal PAB size in bilateral PAB surgery.
Intraoperative Doppler echocardiographic PV-VTI ratio using TEE during
bilateral PAB surgery is effective in predicting the need for reoperation. 